\title{
3 Research Square \\ The Semantics of Natural Objects and Tools in the Brain: A Combined Behavioural and MEG Study
}

\section{Elisa Visani}

Istituto Neurologico Carlo Besta

Davide Rossi Sebastiano

Istituto Neurologico Carlo Besta

\section{Dunja Duran}

Istituto Neurologico Carlo Besta

\section{Gioacchino Garofalo}

Vita-Salute San Raffaele University

\section{Fabio Magliocco}

Azienda Socio-Sanitaria Territoriale di Vimercate

Francesco Silipo

Magna Graecia University

Giovanni Buccino ( $\sim$ buccino.giovanni@hsr.it)

Vita-Salute San Raffaele University

\section{Research Article}

Keywords: Current literature supports, recognition of objects, neural structures, behavioural and MEG results

Posted Date: May 7th, 2021

DOl: https://doi.org/10.21203/rs.3.rs-484130/v1

License: (c) (1) This work is licensed under a Creative Commons Attribution 4.0 International License. Read Full License

Version of Record: A version of this preprint was published at Brain Sciences on January 12th, 2022. See the published version at https://doi.org/10.3390/brainsci12010097. 


\section{Abstract}

Current literature supports the notion that the recognition of objects, when visually presented, is subserved by neural structures different from those responsible for the semantic processing of their nouns. However, embodiment foresees that processing observed objects and their verbal labels should share similar neural mechanisms. In a combined behavioural and MEG study, we compared the modulation of motor responses and cortical rhythms during the processing of graspable natural objects and tools, either verbally or pictorially presented. Our findings demonstrate that conveying meaning to an observed object or processing its noun similarly modulates both motor responses and cortical rhythms; moreover, natural graspable objects and tools affect in a different manner both behavioural and MEG results, independent of presentation modality. These findings provide experimental evidence that neural substrates responsible for conveying meaning to object overlap with those where the object is represented, thus supporting an embodied view of semantic processing.

\section{Introduction}

What is semantics? Classically, semantics refers to our capacity to attribute meaning to the events and the entities (such as objects, words, feelings, and so on) that we experience during lifespan and organize in a symbolic system. Language is the symbolic system that we use to represent this knowledge about the world, but how this knowledge is organized in the brain and how it is related to the real world is a matter of debate within the neuroscientific literature. In recent times, it has been proposed that the speakers understand linguistic material thanks to a recruitment of those sensory, motor and even emotional systems involved in experiencing the content expressed by that linguistic material ${ }^{1-7}$. This approach contrasts with a more classical one, claiming language as an amodal function, that is completely disentangled from those sensorimotor systems normally involved in experiencing its content ${ }^{8-11}$. Indeed, these two approaches are not mutually exclusive, and some authors have tempted to combine the two views. In this perspective, authors do not deny a potential role of sensory, motor and emotional systems in building up concepts, although they claim that the knowledge about objects ad their concepts are stored in brain areas distinct from those where individuals experience the different features of objects ${ }^{12-16}$.

Within this general framework, emphasis has been given to objects and their correspondent verbal labels, nouns. The central claim is that information about the features of an object, such as its form, its size, the manner in which we act upon it, is stored in our sensorial, motor, and emotional systems ${ }^{15}$. For instance, it has been demonstrated that words related to odorants (e.g., cinnamon) activate the olfactory system ${ }^{17}$, words related to taste (e.g., salt) activate the gustatory one ${ }^{18}$ and also words related to emotion activate the corresponding area (e.g., disgust) ${ }^{19}$. However, this evidence does not rule out the possibility that, despite the involvement of the cortex used to experience the sensory or motor content, the mechanism (and possibly the areas) allowing us to attribute the meaning could be different ${ }^{13,15,20}$. The brain regions 
where individuals store object concepts and names seems to be distinct from the regions in which such features are experienced, thus constituting the so-called semantic hubs ${ }^{13,15,21}$.

A further point of interest is that, in current literature, observed objects seems to be differently analysed from their corresponding nouns. There is a general agreement that two visual streams subserve the processing of objects when observed ${ }^{22-24}$. When individuals have to interact with objects, the dorsal stream, including fronto and parietal areas, is mainly involved. This stream is devoted to sensorimotor transformation that make possible the choice of the most appropriate motor program to act upon the observed object. The recognition of object, however, is subserved by the ventral stream including some specific temporal areas (e.g., lateral occipital temporal cortex, anterior and inferotemporal regions). As a further support to this view, clinical findings showed a dissociation between the ability to recognize an object, following a damage in the temporal lobe, and the ability to correct use the object after damage to the parietal cortex ${ }^{25-30}$. Indeed, these two streams are not completely segregated but they rather interact to update our functional knowledge and our capacity to interact online with an object ${ }^{25,31-35}$. To sum up, the neural substrates devoted to object recognition, when visually presented, seem to be distinct from those subserving the semantic of objects when expressed by their verbal referents (i.e., the nouns). Note that the noun of an object is considered to coincide with the concept of the object itself ${ }^{15,36}$.

Tools are a special class of graspable objects for humans. The study of tools is interesting since tools have an associated functional use that involves a particular modality of interaction with the object, rather than just the feature to be grasped, as natural objects have ${ }^{25}$. Furthermore, humans use tools in different contexts, thus requiring a generalization process and the conceptual knowledge of their use 27 . Functional neuroimaging studies focusing on tools have demonstrated that the use of tools elicits an activation of many distinct brain areas, including the left supramarginal gyrus (SMA) ${ }^{37-46}$, the PMv $47-$ ${ }^{49}$, the left inferior frontal gyrus (IFG) bordering pars opercularis ${ }^{27}$, and the left insula ${ }^{38}$. Overall, these studies show that tools are represented in circuits distinct from those when natural objects are represented. Specifically, tools seem to be represented in a fronto-parietal circuits corresponding to ventro-dorsal subdivision of the dorsal pathway ${ }^{50,51}$. Moreover, this different representation is possible already present in non-human primates ${ }^{52}$.

As for natural objects, some recent studies have suggested that the verbal labels and the observed objects share similar semantic mechanisms ${ }^{2,53-59}$. In details, participants provided with slower motor responses for natural graspable objects and nouns as compared to non-graspable ones ${ }^{53,60}$. The authors proposed that when participants are engaged in two different tasks, that is the object processing (either pictorially or verbally presented) and the preparation of motor responses, the motor system is involved in both and therefore there is a competition for neuronal resources, leading to a slowing down of motor responses.

In the present study, we directly compared the modulation of motor system during the processing of natural graspable objects either verbally or pictorially presented, and graspable tools, presented in the 
same modalities. If one accepts the embodiment approach, these two object categories should lead to a different modulation of the motor system, regardless of the presentation modality, since natural graspable objects and tools are differently represented in the brain. On the contrary, if semantics of objects is distinct from their motor representations, then a different modulation could be still potentially found for observed objects but appears unlikely for nouns, since the nouns of objects are stored in specific hubs. In the present study, we have addressed this issue with a go/no-go task already used in previous studies of our group ${ }^{53,61}$ and with Magnetoencephalography (MEG), looking at the modulation of beta rhythm during the semantic processing of natural objects and tools, presented either by nouns or by images. Beta band oscillations are the predominant rhythm originating in the motor cortex with a typical pattern of suppression and rebound during movement ${ }^{62}$. Beta suppression, or desynchronization, starts several hundred milliseconds before movement onset in self-paced or externally cued movements and becomes maximal around the time of movement execution. This suppression was adopted to study

the neural correlates of action observation ${ }^{63,64}$, motor imagery ${ }^{65-67}$ and action related language ${ }^{61,68}$. In the present study this rhythm has been exploited to reveal the neural correlates of object observation and noun processing and the underlying neurophysiological mechanisms of behavioural responses. In this study, behavioural findings have shown that hand-motor responses were slower for natural graspable objects as compared to tools, regardless of presentation modality, and in keeping with this, the modulation of beta rhythm has shown a weaker decrease during the processing of natural stimuli as compared to tools.

\section{Results}

\section{Experiment 1 - Behavioural study}

Data were collected from twenty-eight participants. One participant was excluded from analysis since he/she performed 120 errors. All other participants performed the task well with few errors (5.3\%). Error trials were checked, excluded without replacement and they were not analysed further.

Repeated measures ANOVA ( $r m A N O V A$ ) revealed the main effect of Category $[F(1,26)=99.64 ; \mathrm{MSE}=$ 382.94; $p<0.001]$. Slower RTs were obtained with natural graspable objects as compared with tools ( $714 \pm 11.89 \mathrm{~ms}$ vs. $677 \pm 11.55 \mathrm{~ms}$ ). Responses to natural stimuli were slower than those to tools both for images $[t(26)=6.79, p<0.0001]$ and nouns $[t(26)=5.41, p<0.0001]$. Neither the main effect of Stimulus Type $[F(1,26)=1.57, p=0.221]$ nor the interaction $[F(1,26)=0.76, p=0.390]$ reached the statistical significance. Descriptive statistics are reported in Table 1.

Table 1. Descriptive statistic of behavioural study (Experiment 1). 


\begin{tabular}{|lllllll|}
\hline & Noun & & \multicolumn{5}{c|}{ Image } \\
\hline $\begin{array}{l}\text { Mean } \\
(\mathrm{ms})\end{array}$ & $\begin{array}{l}\text { Standard } \\
\text { deviation }(\mathrm{ms})\end{array}$ & $\begin{array}{l}\text { Standard } \\
\text { error }(\mathrm{ms})\end{array}$ & $\begin{array}{l}\text { Mean } \\
(\mathrm{ms})\end{array}$ & $\begin{array}{l}\text { Standard } \\
\text { deviation }(\mathrm{ms})\end{array}$ & $\begin{array}{l}\text { Standard } \\
\text { error }(\mathrm{ms})\end{array}$ \\
\hline Natural & 708 & 80.91 & 15.57 & 720 & 94.61 & 18.21 \\
\hline Tool & 666 & 76.03 & 14.63 & 686 & 93.27 & 17.95 \\
\hline
\end{tabular}

\section{Experiment 2 - MEG study}

\section{Behavioural data}

Behavioural data from 15 participants replicated the results of Experiment 1. All subjects performed well with few errors (error rate: $4.2 \%$ ). rmANOVA showed a main effect of Category $[F(1,14)=22.18$, MSE $=27093.8, p<0.001]$. RTs to natural stimuli were slower than those to tool stimuli both for images (Natural: $573.3 \pm 11.61 \mathrm{~ms}$; Tools: $536.3 \pm 13.2 \mathrm{~ms}, \mathrm{t}(14)=3.612, \mathrm{p}=0.003$ ) and nouns (Natural: $593.3 \pm 18.9$ ms; Tools: $545.9 \pm 16.3 \mathrm{~ms}, \mathrm{t}(14)=3.877, \mathrm{p}=0.002)$. Neither the main effect of Stimulus Type nor the interaction reached the statistical significance.

\section{MEG data}

\section{Time-frequency analysis on sensors.}

The typical time-frequency pattern was observed in every subject and condition consisting in beta band desynchronization over the contralateral motor area immediately after stimulus onset followed by focal synchronization after movement execution. When comparing natural graspable objects and tools (both for images and nouns), statistical analysis revealed a significant difference in the interval between 0.5 and $1 \mathrm{~s}$ after stimulus onset in contralateral motor area. Specifically, a significant greater desynchronization was found for tools stimuli with respect to natural stimuli (Figure 1.A and 1.B). The difference was greater and more protracted in the case of visual stimuli as compared to nouns (Figure 1.C and 1.D). No significant differences were found in the remaining comparison (natural object images vs. nouns, tool images vs. nouns).

\section{Source analysis}

Cortical sources of beta power modulation by means of dynamic imaging of coherent sources (DICS) are illustrated in Figure 2A. Beta power modulations were most pronounced in contralateral pericentral regions, including pre-central, post-central and inferior parietal areas. Comparing the beta desynchronization in the area under the curve (AUC) of selected region of interest (ROI), rmANOVA showed a main effect of Category (contralateral precentral area: $F(1,14)=7.141, p=0.018$; contralateral precentral area: $F(1,14)=13.785, p=0.002$; contralateral inferior parietal lobule: $F(1,14)=5.158, p=0.039$. On average tools stimuli showed greater desynchronization AUC than natural stimuli, reaching the 
significance for the images in precentral $[t(14)=-3.279, p=0.005]$ and postcentral $[t(14)=-3.597, p=0.003]$ areas (Figure 2B and 2C).

\section{Discussion}

The results of the present study are relevant for the current literature about the semantics of objects. First element of interest is the evidence that conveying meaning to an observed object or processing the verbal label of an object (i.e., noun) similarly modulates the activity of the motor system as reflected in motor responses and in the modulation of the beta rhythm known to be generated in frontal and parietal areas, as revealed by MEG. For natural objects participants gave slower motor responses as compared to tools regardless the presentation modality. The same pattern of motor responses has been found in a previous study ${ }^{53}$, when comparing seen and verbally labelled graspable and non-graspable objects, with a slowing down of RTs with graspable objects as compared to non-graspable ones, also in this case regardless of presentation modality. This interference effect has been explained assuming that the same neural resources (namely the same sectors of the motor system) were involved in giving motor responses and judging the presented language material. Hence, participants paid a cost showing a slowing down of their motor responses. In the present experiment, the interference effect was replicated in the MEG study, where participants were requested to perform the same go/no-go task while assessing the cortical rhythms. Motor responses to natural graspable objects confirmed to be slower than those given to tools, thus further supporting the notion that tools and natural graspable objects have a different representation within the motor system. Coherently, beta rhythm, as revealed by MEG, had a weaker decrease during the processing of natural graspable objects as compared to tools. A suppression of beta rhythm, normally recorded in motor/premotor areas, occurs when these areas are involved in actual execution of an action or, at a less degree, when individuals observe or imagine an action ${ }^{62,69}$. In other words, our results show that, during the processing of natural stimuli, the suppression of beta rhythm is weaker than during the processing of tools, thus suggesting that the motor system is less prompt to give a motor response. This weaker suppression appears the neurophysiological correlate of the interference effect obtained in the behavioural task.

It is worth stressing that converging results also come from very few fMRI studies showing shared neural substrates activation during the processing of nouns and visually presented objects ${ }^{70-72}$, thus supporting further the view of a common semantic system for both nouns and their corresponding objects ${ }^{73-75}$. Similar results were obtained during behavioural, neurophysiological and MEG studies where participants were asked to process observed hand-actions and verbs expressing actions in the same category, either taken separately or combined ${ }^{61,64,76-81}$.

As far as observed natural objects, the present results are in keeping with the current literature $2,15,21,22$, showing that the dorsal stream is involved when participants observe natural graspable objects, as the relevant features of these objects are the motor ones. However, the present results show that a similar modulation of motor responses and beta rhythm occur also for verbal labels referring to the same object 
category, thus suggesting that the dorsal stream was similarly involved independent of presentation modality. This evidence does not fit with current view claiming that the conceptual knowledge about an object is represented in semantic hubs distinct from the brain areas where object properties are coded $13,15,21$. These areas widely coincide with posterior inferior parietal lobule (including the angular gyrus, $\mathrm{IPL}$ ), middle temporal gyrus, fusiform and parahippocampal gyri, dorsomedial prefrontal cortex, inferior frontal gyrus, ventromedial prefrontal cortex, and posterior cingulate gyrus ${ }^{13,20}$.

The unexpected recruitment of the dorsal stream during the processing of natural graspable object nouns in the present experiment could reflect the spread of activity of top-down cognitive processes most likely occurring in higher order areas involved in object identification ${ }^{9}$. We tend to rule out this explanation, since processing of our stimuli is time locked at $150 \mathrm{~ms}$ from stimulus presentation, a time window which rules out the occurrence of motor system recruitment as a side effect of upstream cognitive processes ${ }^{76,77,79,81-84}$.

A second point of interest is the evidence that observed graspable tools and nouns referring to this object category do not modulate the activity of the motor system in the same manner as natural objects do. As we stated in the introduction, tools are a special class of graspable objects that imply special hand-object interactions. Manipulation of tools is mainly devoted to a specific use rather than to an "ecological" grasping. By ecological grasping, in this context, we refer to grasping actions that we can act upon natural objects and that are likely shared also with other species, even phylogenetically far from human primates. For example, picking up a nut is an action shared with individuals of other species, and possibly similarly represented in the brain.

Despite there is a general debate on the use of tools in monkeys ${ }^{85,86}$ there is no doubt that only humans possess specialized neural mechanisms allowing them to understand the functional properties of tools. Moreover, only humans have the capacity to generalize the use of a tool in different contexts and to build up new tools depending on their needs. A so fine developed ability seems to have its neural basis in the left IPL that appears as a specific sector only evolved in homo-sapiens, distinct from monkey grasping regions 52,87 . Within the dorsal stream, this area is referred to as ventro-dorsal sector ${ }^{25,50,51,88}$. A further consideration that supports the notion that the use of tools is exclusive for humans comes from clinical neurology. Apraxia is a syndrome where patients may lose the capacity to use tools properly ${ }^{89-91}$. Apparently, there is no counterpart of apraxia syndrome in the monkeys ${ }^{92}$. If one accepts the notion that the semantic of objects is coded where the objects are represented, then processing tools should imply the involvement of the corresponding brain sector in the ventro-dorsal circuit. The results of the present study are in line with this view. Tools, whatever the modality of presentation, did not modulate the motor responses as well as beta rhythm, like natural objects did. This evidence may be explained by the fact that participants used a very simple motor act to provide the responses (pushing a button), an action represented in the circuit devoted to interactions with natural objects rather than in the circuit devoted to the use of tools. A similar distinction was revealed by using TMS ${ }^{93}$ in a study where motor evoked potentials (MEPs) were obtained during the observation of graspable and non-graspable natural objects 
and tools, respectively. Results showed that MEPs elicited by natural graspable objects had a less amplitude than those elicited by graspable tools, again suggesting that a different circuit and a different sector of premotor/motor cortex was involved in processing these two categories of objects.

One could argue that tools nouns did not affect motor responses and beta rhythms in the present experiment because, as foreseen by current literature, nouns are processed in semantic hubs. However, if one assumes that nouns are coded in specific semantic hubs, then the nouns of tools as well as the nouns of natural objects should be coded in these semantic hubs and, consequently, should not modulate the activity of motor areas. In other words, one should expect similar motor responses as well as a similar modulation of beta rhythm when processing nouns referring both to natural graspable objects and tools. The present data, showing that only nouns of natural graspable objects modulate the activity of areas devoted to ecological grasping, further support the notion that the neural substrates of semantics processing overlap with those where the most relevant features of an object are experienced.

If semantics is coded in the areas where objects are motorically represented or perceptually experienced, then it remains to explain the role of the higher order areas that several authors consider as the actual semantic hubs $13,21,94,95$. Beyond language processing, these areas have been involved in different tasks. Some of them also constitute the nodes of the so-called "default-mode" network, a set of functionally interconnected regions that are consistently modulated during demanding cognitive tasks ${ }^{96,97}$ or during social cognition tasks ${ }^{98-100}$. As for prefrontal cortex areas, they have been involved in working memory tasks ${ }^{101}$ as well as in the re-organization of simple and well-known motor acts in novel actions ${ }^{102,103}$. Finally, the inferior frontal gyrus (i.e., the Broca's region) is known to be endowed with hand motor representations and has a role in speech production as well as lip reading ${ }^{104-106}$. We forward that the recruitment of these areas during nouns processing and conceptualization, rather than related to semantics, is better explained if we assume that they may contribute to contextualize the processed words, to express how demanding is their processing and, most likely, how much they are related to our life experiences and personal beliefs.

\section{Methods}

\section{Behavioural study}

\section{Participants}

Twenty-eight volunteers ( 18 females, age $=22.4 \pm 3.2$ years) took part in the behavioural experiment. All participants were 18 years or older prior to participating, they gave their informed consent, accordingly with the ethical standards of the Declaration of Helsinki. Exclusion criteria were formal education in linguistics, the presence of neurological or psychiatric disorders and the use of drugs affecting the central nervous system. The study was approved by the Ethics Committee of the University "Magna Graecia" of Catanzaro and complied with the ethical standards of the Italian Psychological Society (AIP, see http://www.aipass.org/node/26) as well as the Italian Board of Psychologists (see 
http://www.psy.it/codice_deontologico.html). All participants were right-handed, according to the Edinburgh Handedness Inventory ${ }^{107}$, had normal or corrected-to-normal vision and were native Italian speakers.

\section{Apparatus, procedure and stimuli}

The experiment was carried out in a sound-attenuated room, dimly illuminated by a halogen lamp directed toward the ceiling. Participants sat comfortably in front of a PC screen (LG 22" LCD, $1920 \times 1080$ pixel resolution and $60 \mathrm{~Hz}$ refresh rate). The eye-to-screen distance was set at $60 \mathrm{~cm}$.

The experiment used a go/no-go task, in which participants were requested to respond to real nouns and images of objects and refrain from responding when presented stimuli were pseudowords and scrambled images. The experiment session consisted of 1 practice block and 1 experimental block. In the practice block, participants were presented with 16 stimuli (4 images of natural objects or tools, 4 scrambled images, 4 nouns of natural objects or tools and 4 pseudowords) which were not used in the experimental block. During the practice block, participants received feedback ("ERROR") after giving a wrong response (i.e., responding to a meaningless or refraining from responding to a real item), as well as for responses given prior to go signal presentation ("ANTICIPATION"), or later than $1.5 \mathrm{~s}$ ("YOU HAVE NOT ANSWERED"). In the experimental block each stimulus was randomly presented twice, for a total of 320 trials, with the constraint that no more than three items of the same kind (verbal, visual) or referring to objects of the same category (graspable natural object, tools, meaningless) could be presented on consecutive trials. No feedback was given to participants. Thus, the experiment, which lasted about 20 min, consisted of 160 go trials ( 80 nouns, $50 \%$ natural graspable object nouns and $50 \%$ tools nouns, plus 80 images of objects, $50 \%$ natural graspable objects and $50 \%$ tools) and 160 no-go trials ( 80 pseudowords plus 80 scrambled images), and 16 practice trials, for a total of 336 trials. To sum up, the experiment used a $2 \times 2$ repeated measures factorial design with Category (natural graspable objects, graspable tools) and Stimulus Type (nouns, photos) as within-subjects variables.

Nouns in the 2 categories were matched for word length (mean values for nouns referring to natural objects and tools: 6.4 and $7.4 ; t=0.049, p=0.96$ ), syllable number (mean values: 2.45 and $3.00 ; t=0.018$, $p=0.98$ ) and written lexical frequency [mean values: 6.14 and 8.77 number of occurrences per million in CoLFIS (Corpus e Lessico di Frequenza dell'Italiano Scritto 3.798.000 words) - Laudanna et al., 1995; $t$ $=0.52, p=0.60]$. Pseudowords were built by substituting one consonant and one vowel in two distinct syllables of each noun (e.g., "sgalpillo" instead of "scalpello"). With this procedure, pseudowords contained orthographically and phonologically legal syllables for the Italian language. Hence, nouns and pseudowords were also matched for length.

Images depicted 20 natural graspable objects and 20 tools. They were photos of real objects and not sketches. The scrambled images were built by applying Photoshop distorting graphic filters (e.g., blur and twist) to the photos depicting both natural graspable objects and graspable tools so to make them unrecognizable and then meaningless. All photos and scrambled images were $440 \times 440$ pixels. 
Each trial started with a black fixation cross ( $R G B$ coordinates $=0,0,0)$ displayed at the center of a grey background (RGB coordinates $=178,178,178$ ). After a delay of $1000-1500 \mathrm{~ms}$ (in order to avoid response habituation), the fixation cross was replaced by a stimulus item, either a noun/pseudoword or an image/scrambled image. Note that the delay could be at any time between 1000 and $1500 \mathrm{~ms}$. The verbal labels were written in black lowercase Courier New bold (font size $=24)$. Stimuli were centrally displayed and surrounded by a red (RGB coordinates $=255,0,0) 20$ pixels-wide frame. The red frame changed to green (RGB coordinates $=0,255,0) 150 \mathrm{~ms}$ after the stimulus onset. The color change of the frame was the "go" signal for the response (Fig.3). Participants were instructed to give a motor response, as fast and accurate as possible, by pressing a key on a computer keyboard centred on participants' body midline with their right index finger. They had to respond when the stimulus referred to a real object, and refrain from responding when it was meaningless. After the go signal, stimuli remained visible for 1350 ms or until participant's responses. Stimulus presentation and response times (RTs) collection were controlled using the software package E-Prime 2.

\section{Data analysis}

Data analyses were performed using R 3.6.3 ${ }^{108}$. Practice trials were excluded from analysis. Participants' RTs to real stimuli were analysed. The RTs were measured from the "go" signal to the button pressing. Mean RTs of each participants were submitted to an rmANOVA, with Category (2 levels: natural graspable object and tool) and Stimulus type (2 levels: noun and image) as factors, with the Greenhouse-Geisser correction being applied in the case of a violation of sphericity assumptions.

\section{MEG study}

\section{Participants}

Fifteen volunteers ( 9 females, age $26.5 \pm 2.0$ years) were recruited for the experiment. All participants were 18 years or older prior to participating. All participants were right-handed, according to the Edinburgh Handedness Inventory ${ }^{107}$, had normal or corrected-to-normal vision and were native Italian speakers. Exclusion criteria were formal education in linguistics, the presence of neurological or psychiatric disorders and the use of drugs affecting the central nervous system. The experiment was carried out in accordance with the ethical standards laid down in the 1964 Declaration of Helsinki and its later amendments. The study was approved by the Ethics Committee of Fondazione IRCCS Istituto Neurologico Carlo Besta of Milan and the University "Magna Graecia" of Catanzaro and complied with the ethical standards of the Italian Psychological Society (AIP, see http://www.aipass.org/node/26) as well as the Italian Board of Psychologists (see http://www.psy.it/codice_deontologico.html). Participants gave their written informed consent before being included in the study.

\section{Task}

Stimuli and procedure were the same of the behavioural study, with the necessary adaptation required by the MEG setting used in the current study. Sixteen practice trials were used to train participants. To 
improve signal-to-noise ratio, the experiment consisted of two consecutive acquisitions in which 80 go trials ( 40 nouns, $50 \%$ natural object nouns and $50 \%$ tools nouns, plus 40 images of object, $50 \%$ natural objects and $50 \%$ tools) and 80 no-go trials ( 40 pseudowords plus 40 scrambled images) were presented, for a total of 320 experimental trials. In the two acquisitions, the presentation order of the stimuli was randomized. Hence, the MEG study used the same $2 \times 2$ repeated measures factorial design as the behavioural one. Stimulus presentation and RTs collection were controlled using the software package Stim2.

\section{MEG data acquisition and pre-processing}

The MEG signals were acquired using a 306-channel whole head MEG system (Triux, Elekta Oy, Helsinki, Finland). Surface EMG signals were simultaneously recorded from pairs of electrodes placed bilaterally

2-3 cm apart over the belly of the right and left flexor and extensor of wrist. Signals were sampled at 1 $\mathrm{kHz}$. Also bipolar electro-oculographic and electrocardiographic signals were acquired.

The participant's head position inside the MEG helmet was continuously monitored by five head position identification (HPI) coils located on the scalp. The locations of these coils, together with three anatomical landmarks (nasion, right and left preauriculars), and additional scalp points were digitized before the recording by means of a 3D digitizer (FASTRAK, Polhemus, Colchester, VT).

The raw MEG data were pre-processed off-line using the spatio-temporal signal-space separation method $^{109}$ implemented in the Maxfilter 2.2 (Elekta Neuromag Oy, Helsinki, Finland) in order to subtract external interference and correct for head movements and then band-pass filtered at $0.1-100 \mathrm{~Hz}$.

Cardiac and ocular movement artifacts were removed using ICA algorithm based on EEGLAB toolbox ${ }^{110}$ implemented in a custom-made MATLAB code (R2017b, Mathworks Inc., Natick MA, USA). MEG data were divided into epochs ranging from $2.2 \mathrm{~s}$ before to $2.8 \mathrm{~s}$ after the stimulus onset. Epochs with muscular artifacts and/or sensor jumps were excluded from further analysis. Finally, data epochs were grouped according to the four conditions (natural and tools images, natural and tools words).

\section{Sensors analysis}

Time-frequency representations (TFR) for frequencies between 15 and $30 \mathrm{~Hz}$ with steps of $1 \mathrm{~Hz}$ were computed using a Fourier transformation. Desynchronization values were obtained as percent power change in beta band $(15-30 \mathrm{~Hz})$ calculated with respect to mean power in the -3 to $-2 \mathrm{~s}$ before cue onset. Finally, for each participant, the most reactive $\beta$-band frequency (individual reactive frequency, IRF) was defined as the frequency at which the maximum desynchronization was found.

\section{Source analysis}

Dynamic imaging of coherent sources (DICS) beamforming ${ }^{111}$ was used to identify the spatial distribution in the frequency domain. The leadfield matrix was computed using realistically shaped single-shell volume conduction model based on template brain co-registered by means of digitized scalp 
points. Source model was obtained from a $5 \mathrm{~mm}$ resolution grid which covered whole brain volume. Source localizations was performed for the band IRF $\pm 1 \mathrm{~Hz}$ for a pre-stimulus baseline period $(-1.2$ to $-0.5 \mathrm{~s}$ ) and for a window of interest during stimulus presentation ( 0.5 to $1.2 \mathrm{~s}$ ) using a common spatial filter based on the pooled data from both time intervals. Subject-specific relative power differences were grand-averaged and visualized on the cortical surface of the MNI brain. To obtain a time course for each trial and voxel, we used the linearly constraint minimum variance (LCMV) beamforming ${ }^{112}$ calculating the covariance matrix of the sensor-level MEG data with $5 \%$ regularization.

Automated Anatomical Labelling atlas was used to extract the source time-series on inferior parietal lobule and precentral and postcentral areas. Subsequently, as for the sensors data, we calculated the desynchronization in IRF $\pm 1 \mathrm{~Hz}$ band and averaged within regions. Finally, we calculated AUC in the 0.5 to $1.5 \mathrm{~s}$ period.

Both sensor and source data analysis were analysed using custom Matlab (MATLAB 2017a, MathWorks, Inc., Natick, MA, USA) scripts based on SPM8 and Fieldtrip toolboxes ${ }^{113,114}$.

\section{Statistical analysis}

The RTs and source time-series AUC were compared using rmANOVA with the factor Category (tools, natural) and Stimulus type (images, nouns) with the Greenhouse-Geisser correction being applied in the case of a violation of sphericity assumptions.

To compare TFR between different conditions in contralateral motor areas, and to identify significant beta frequencies and time points, the non-parametric permutation test in combination with cluster-level statistics and multiple comparison correction implemented in Fieldtrip toolbox was applied. Post-hoc paired two-tailed t tests were used to calculate the within-group difference between stimuli. All data are expressed as mean \pm standard errors of mean. Statistical analyses were carried out using IBM SPSS, version 20 (SPSS Inc., Chicago, IL, U.S.A.).

\section{Declarations}

\section{Author Contributions}

G.B. conceived and designed the study. F.M. and F.S. selected and prepared stimuli. E.V. and D.D. implemented the task and analysis code for experiment 2. E.V., D.D. and D.R.S. collected MEG data. F.M. and F.S. collected behavioural data for experiment 1. E.V. analysed behavioural and MEG data for experiment 2. G.G. analysed behavioural data of experiment 1. G.B., D.R.S., G.G . and E.V. wrote the manuscript. G.B. and D.R.S provided with critical revision. All authors discussed the results and commented on manuscript.

\section{Competing interest statement}

The authors declare no competing interests. 


\section{References}

1. Barsalou, L. W. Grounded cognition. Annu. Rev. Psychol.59, 617-645 (2008).

2. Buccino, G., Colagè, I., Gobbi, N. \& Bonaccorso, G. Grounding meaning in experience: A broad perspective on embodied language. Neurosci. Biobehav. Rev.69, 69-78 (2016).

3. Fischer, M. H. \& Zwaan, R. A. Embodied language: A review of the role of the motor system in language comprehension. Q. J. Exp. Psychol.61, 825-850 (2008).

4. Gallese, V. Mirror neurons and the social nature of language: The neural exploitation hypothesis. Soc. Neurosci.3, 317-333 (2008).

5. Pulvermüller, F. A brain perspective on language mechanisms: from discrete neuronal ensembles to serial order. Prog. Neurobiol.67, 85-111 (2002).

6. Vigliocco, G. et al. The Neural Representation of Abstract Words: The Role of Emotion. Cereb. Cortex24, 1767-1777 (2014).

7. Kousta, S. T., Vigliocco, G., Vinson, D. P., Andrews, M. \& Del Campo, E. The Representation of Abstract Words: Why Emotion Matters. J. Exp. Psychol. Gen.140, 14-34 (2011).

8. Chatterjee, A. Disembodying cognition. Lang. Cogn.2, 79-116 (2011).

9. Mahon, B. Z. \& Caramazza, A. A critical look at the embodied cognition hypothesis and a new proposal for grounding conceptual content. J. Physiol. Paris102, 59-70 (2008).

10. Mahon, B. Z. \& Caramazza, A. The orchestration of the sensory-motor systems: Clues from neuropsychology. Cogn. Neuropsychol.22, 480-494 (2005).

11. Pylyshyn, Z. Return of the mental image: Are there really pictures in the brain? Trends Cogn. Sci.7, 113-118 (2003).

12. Desai, R. H., Binder, J. R., Conant, L. L. \& Seidenberg, M. S. Activation of Sensory-Motor Areas in Sentence Comprehension. Cereb. Cortex20, 468-478 (2010).

13. Fernandino, L. et al. Concept Representation Reflects Multimodal Abstraction: A Framework for Embodied Semantics. Cereb. Cortex26, 2018-2034 (2016).

14. Mahon, B. Z. \& Kemmerer, D. Interactions between language, thought, and perception: Cognitive and neural perspectives. Cogn. Neuropsychol.37, 235-240 (2020).

15. Martin, A. GRAPES-Grounding representations in action, perception, and emotion systems: How object properties and categories are represented in the human brain. Psychon. Bull. Rev.23, 979-990 (2016).

16. Martin, A. The representation of object concepts in the brain. Annu. Rev. Psychol.58, 25-45 (2007).

17. González, J. et al. Reading cinnamon activates olfactory brain regions. Neuroimage32, 906-912 (2006).

18. Barrós-Loscertales, A. et al. Reading salt activates gustatory brain regions: FMRI evidence for semantic grounding in a novel sensory modality. Cereb. Cortex22, 2554-2563 (2012). 
19. Ponz, A. et al. Emotion processing in words: A test of the neural re-use hypothesis using surface and intracranial EEG. Soc. Cogn. Affect. Neurosci.9, 619-627 (2014).

20. Binder, J. R., Desai, R. H., Graves, W. W. \& Conant, L. L. Where is the semantic system? A critical review and meta-analysis of 120 functional neuroimaging studies. Cereb. Cortex19, 2767-2796 (2009).

21. Mahon, B. Z. What is embodied about cognition? Lang. Cogn. Neurosci.30, 420-429 (2015).

22. Milner, A. D. \& Goodale, M. A. Two visual systems re-viewed. Neuropsychologia46, 774-785 (2008).

23. Goodale, M. A. \& Milner, A. D. Two visual streams: Interconnections do not imply duplication of function. Cogn. Neurosci.1, 65-68 (2010).

24. Goodale, M. A. \& Milner, A. D. Separate visual pathways for perception and action. Trends Neurosci.15, 20-25 (1992).

25. Binkofski, F. \& Buxbaum, L. J. Two action systems in the human brain. Brain Lang.127, 222-229 (2013).

26. Kalénine, S., Buxbaum, L. J. \& Coslett, H. B. Critical brain regions for action recognition: Lesion symptom mapping in left hemisphere stroke. Brain133, 3269-3280 (2010).

27. Johnson-Frey, S. H. The neural bases of complex tool use in humans. Trends Cogn. Sci.8, 71-78 (2004).

28. Hodges, J. R., Spatt, J. \& Patterson, K. 'What' and 'how': Evidence for the dissociation of object knowledge and mechanical problem-solving skills in the human brain. Proc. Natl. Acad. Sci. U. S. A.96, 9444-9448 (1999).

29. Negri, G. A. L. et al. What is the role of motor simulation in action and object recognition? Evidence from apraxia. Cogn. Neuropsychol.24, 795-816 (2007).

30. Gonzalez Rothi, L. J., Ochipa, C. \& Heilman, K. M. A Cognitive Neuropsychological Model of Limb Praxis. Cogn. Neuropsychol.8, 443-458 (1991).

31. van Polanen, V. \& Davare, M. Interactions between dorsal and ventral streams for controlling skilled grasp. Neuropsychologia79, 186-191 (2015).

32. Cohen, N. R., Cross, E. S., Tunik, E., Grafton, S. T. \& Culham, J. C. Ventral and dorsal stream contributions to the online control of immediate and delayed grasping: A TMS approach. Neuropsychologia47, 1553-1562 (2009).

33. Uccelli, S., Pisu, V., Riggio, L. \& Bruno, N. The Uznadze illusion reveals similar effects of relative size on perception and action. Exp. Brain Res.237, 953-965 (2019).

34. Kopiske, K. K., Bruno, N., Hesse, C., Schenk, T. \& Franz, V. H. The functional subdivision of the visual brain: Is there a real illusion effect on action? A multi-lab replication study. Cortex79, 130-152 (2016).

35. Whitwell, R. L., Milner, A. D. \& Goodale, M. A. The two visual systems hypothesis: New challenges and insights from visual form agnosic patient DF. Front. Neurol.5, 1-8 (2014). 
36. Rosch, E., Mervis, C. B., Gray, W. D., Johnson, D. M. \& Boyes-Braem, P. Basic objects in natural categories. Cogn. Psychol.8, 382-439 (1976).

37. Boronat, C. B. et al. Distinctions between manipulation and function knowledge of objects: evidence from functional magnetic resonance imaging. Cogn. Brain Res.23, 361-373 (2005).

38. Brandi, M.-L., Wohlschläger, A., Sorg, C. \& Hermsdörfer, J. The Neural Correlates of Planning and Executing Actual Tool Use. J. Neurosci.34, 13183 LP - 13194 (2014).

39. Buchwald, M., Przybylski, Ł. \& Króliczak, G. Decoding Brain States for Planning Functional Grasps of Tools: A Functional Magnetic Resonance Imaging Multivoxel Pattern Analysis Study. J. Int. Neuropsychol. Soc.24, 1013-1025 (2018).

40. Marques, J. F., Canessa, N. \& Cappa, S. Neural differences in the processing of true and false sentences: Insights into the nature of 'truth' in language comprehension. Cortex45, 759-768 (2009).

41. Chen, Q., Garcea, F. E., Jacobs, R. A. \& Mahon, B. Z. Abstract Representations of Object-Directed Action in the Left Inferior Parietal Lobule. Cereb. Cortex28, 2162-2174 (2018).

42. Chen, Q., Garcea, F. E. \& Mahon, B. Z. The Representation of Object-Directed Action and Function Knowledge in the Human Brain. Cereb. Cortex26, 1609-1618 (2016).

43. Gallivan, J. P., Adam McLean, D., Valyear, K. F. \& Culham, J. C. Decoding the neural mechanisms of human tool use. Elife2013, 1-29 (2013).

44. Hermsdörfer, J., Terlinden, G., Mühlau, M., Goldenberg, G. \& Wohlschläger, A. M. Neural representations of pantomimed and actual tool use: Evidence from an event-related fMRI study. Neuroimage36, T109-T118 (2007).

45. Kellenbach, M. L., Brett, M. \& Patterson, K. Actions Speak Louder Than Functions: The Importance of Manipulability and Action in Tool Representation. J. Cogn. Neurosci.15, 30-46 (2003).

46. Rumiati, R. I. et al. Neural basis of pantomiming the use of visually presented objects. Neuroimage21, 1224-1231 (2004).

47. Chao, L. L. \& Martin, A. Representation of manipulable man-made objects in the dorsal stream. Neuroimage12, 478-484 (2000).

48. Grafton, S. T., Fadiga, L., Arbib, M. A. \& Rizzolatti, G. Premotor cortex activation during observation and naming of familiar tools. Neuroimage6, 231-236 (1997).

49. Króliczak, G. \& Frey, S. H. A Common Network in the Left Cerebral Hemisphere Represents Planning of Tool Use Pantomimes and Familiar Intransitive Gestures at the Hand-Independent Level. Cereb. Cortex19, 2396-2410 (2009).

50. Rizzolatti, G. \& Matelli, M. Two different streams form the dorsal visual system: Anatomy and functions. Exp. Brain Res.153, 146-157 (2003).

51. Sakreida, K. et al. Affordance processing in segregated parieto-frontal dorsal stream sub-pathways. Neurosci. Biobehav. Rev.69, 89-112 (2016).

52. Peeters, R. et al. The Representation of Tool Use in Humans and Monkeys: Common and Uniquely Human Features. J. Neurosci.29, 11523 LP - 11539 (2009). 
53. Marino, B. F. M. et al. Viewing photos and reading nouns of natural graspable objects similarly modulate motor responses. Front. Hum. Neurosci.8, 1-10 (2014).

54. Vigliocco, G., Vinson, D. P., Druks, J., Barber, H. \& Cappa, S. F. Nouns and verbs in the brain: A review of behavioural, electrophysiological, neuropsychological and imaging studies. Neurosci. Biobehav. Rev.35, 407-426 (2011).

55. Harpaintner, M., Sim, E. J., Trumpp, N. M., Ulrich, M. \& Kiefer, M. The grounding of abstract concepts in the motor and visual system: An fMRI study. Cortex124, 1-22 (2020).

56. Buccino, G. et al. Processing graspable object images and their nouns is impaired in Parkinson's disease patients. Cortex100, 32-39 (2018).

57. Horoufchin, H., Bzdok, D., Buccino, G., Borghi, A. M. \& Binkofski, F. Action and object words are differentially anchored in the sensory motor system - A perspective on cognitive embodiment. Sci. Rep.8, 1-11 (2018).

58. Zhang, Z., Sun, Y. \& Humphreys, G. W. Perceiving object affordances through visual and linguistic pathways: A comparative study. Sci. Rep.6, 1-9 (2016).

59. Bub, D. N., Masson, M. E. J. \& Kumar, R. Time course of motor affordances evoked by pictured objects and words. J. Exp. Psychol. Hum. Percept. Perform.44, 53-68 (2018).

60. Buccino, G., Marino, B. F., Bulgarelli, C. \& Mezzadri, M. Fluent speakers of a second language process graspable nouns expressed in L2 like in their native language. Front. Psychol.8, 1-8 (2017).

61. Klepp, A., Niccolai, V., Buccino, G., Schnitzler, A. \& Biermann-Ruben, K. Language-motor interference reflected in MEG beta oscillations. Neuroimage109, 438-448 (2015).

62. Pfurtscheller, G. \& Lopes da Silva, F. H. Event-related EEG/MEG synchronization and desynchronization: basic principles. Clin. Neurophysiol.110, 1842-1857 (1999).

63. Hari, R. \& Kujala, M. V. Brain basis of human social interaction: From concepts to brain imaging. Physiol. Rev.89, 453-479 (2009).

64. Moreno, I., de Vega, M. \& León, I. Understanding action language modulates oscillatory mu and beta rhythms in the same way as observing actions. Brain Cogn.82, 236-242 (2013).

65. Schnitzler, A., Salenius, S., Salmelin, R., Jousmäki, V. \& Hari, R. Involvement of Primary Motor Cortex in Motor Imagery: A Neuromagnetic Study. Neuroimage6, 201-208 (1997).

66. de Lange, F. P., Roelofs, K. \& Toni, I. Motor imagery: A window into the mechanisms and alterations of the motor system. Cortex44, 494-506 (2008).

67. Brinkman, L., Stolk, A., Dijkerman, H. C., de Lange, F. P. \& Toni, I. Distinct Roles for Alpha- and BetaBand Oscillations during Mental Simulation of Goal-Directed Actions. J. Neurosci.34, 14783 LP 14792 (2014).

68. Weiss, S. \& Mueller, H. "Too Many betas do not Spoil the Broth": The Role of Beta Brain Oscillations in Language Processing. Frontiers in Psychology vol. 3201 (2012).

69. Hari, R. et al. Activation of human primary motor cortex during action observation: A neuromagnetic study. Proc. Natl. Acad. Sci.95, 15061 LP - 15065 (1998). 
70. Shinkareva, S. V., Malave, V. L., Mason, R. A., Mitchell, T. M. \& Just, M. A. Commonality of neural representations of words and pictures. Neuroimage54, 2418-2425 (2011).

71. Devereux, B. J., Clarke, A., Marouchos, A. \& Tyler, L. K. Representational similarity analysis reveals commonalities and differences in the semantic processing of words and objects. J. Neurosci.33, 18906-18916 (2013).

72. Simanova, I., Hagoort, P., Oostenveld, R. \& Van Gerven, M. A. J. Modality-independent decoding of semantic information from the human brain. Cereb. Cortex24, 426-434 (2014).

73. Vandenberghe, R., Price, C., Wise, R., Josephs, O. \& Frackowiak, R. S. J. Functional anatomy of a common semantic system for words and pictures. Nature vol. 383 254-256 (1996).

74. Ganis, G., Kutas, M. \& Sereno, M. I. The Search for “Common Sense”: An Electrophysiological Study of the Comprehension of Words and Pictures in Reading. J. Cogn. Neurosci.8, 89-106 (1996).

75. Van Doren, L., Dupont, P., De Grauwe, S., Peeters, R. \& Vandenberghe, R. The amodal system for conscious word and picture identification in the absence of a semantic task. Neuroimage49, 32953307 (2010).

76. Sato, M., Mengarelli, M., Riggio, L., Gallese, V. \& Buccino, G. Task related modulation of the motor system during language processing. Brain Lang.105, 83-90 (2008).

77. Buccino, G. et al. Listening to action-related sentences modulates the activity of the motor system: A combined TMS and behavioral study. Cogn. Brain Res.24, 355-363 (2005).

78. de Vega, M., Moreno, V. \& Castillo, D. The comprehension of action-related sentences may cause interference rather than facilitation on matching actions. Psychol. Res.77, 20-30 (2013).

79. Santana, E. J. \& De Vega, M. An ERP study of motor compatibility effects in action language. Brain Res.1526, 71-83 (2013).

80. Dalla Volta, R., Gianelli, C., Campione, G. C. \& Gentilucci, M. Action word understanding and overt motor behavior. Exp. Brain Res.196, 403-412 (2009).

81. Boulenger, V. et al. Cross-talk between language processes and overt motor behavior in the first 200 msec of processing. J. Cogn. Neurosci.18, 1607-1615 (2006).

82. Chersi, F., Thill, S., Ziemke, T. \& Borghi, A. M. Sentence processing: Linking language to motor chains. Front. Neurorobot.4, 1-9 (2010).

83. Marino, B. F. M., Gough, P. M., Gallese, V., Riggio, L. \& Buccino, G. How the motor system handles nouns: a behavioral study. Psychol. Res.77, 64-73 (2013).

84. García, A. M. \& Ibáñez, A. A touch with words: Dynamic synergies between manual actions and language. Neurosci. Biobehav. Rev.68, 59-95 (2016).

85. Maravita, A. \& Iriki, A. Tools for the body (schema). Trends Cogn. Sci.8, 79-86 (2004).

86. Iriki, A., Tanaka, M. \& Iwamura, Y. Coding of modified body schema during tool use by macaque postcentral neurones. Neuroreport7, (1996).

87. Nelissen, K. \& Vanduffel, W. Grasping-related functional MRI brain responses in the macaque monkey. J. Neurosci.31, 8220-8229 (2011). 
88. Errante, A., Ziccarelli, S., Mingolla, G. \& Fogassi, L. Grasping and Manipulation: Neural Bases and Anatomical Circuitry in Humans. Neuroscience458, 203-212 (2021).

89. De Renzi, E. \& Lucchelli, F. IDEATIONAL APRAXIA. Brain111, 1173-1185 (1988).

90. Heilman, K. M., Schwartz, H. D. \& Geschwind, N. Defective motor learning in ideomotor apraxia. Neurology25, 1018 LP - 1018 (1975).

91. Buxbaum, L. J., Veramontil, T. \& Schwartz, M. F. Function and manipulation tool knowledge in apraxia: Knowing 'what for' but not 'how'. Neurocase6, 83-97 (2000).

92. Caminiti, R. et al. Understanding the parietal lobe syndrome from a neurophysiological and evolutionary perspective. Eur. J. Neurosci.31, 2320-2340 (2010).

93. Gough, P. M. et al. Nouns referring to tools and natural objects differentially modulate the motor system. Neuropsychologia50, 19-25 (2012).

94. Desai, R. H., Herter, T., Riccardi, N., Rorden, C. \& Fridriksson, J. Concepts within reach: Action performance predicts action language processing in stroke. Neuropsychologia71, 217-224 (2015).

95. Martin, A. \& Martin, A. GRAPES - Grounding representations in action, perception , and emotion systems: How object properties and categories are represented in the human brain. Psychon. Bull. Rev. 979-990 (2016) doi:10.3758/s13423-015-0842-3.

96. Raichle, M. E. et al. A default mode of brain function. Proc. Natl. Acad. Sci. U. S. A.98, 676-682 (2001).

97. Raichle, M. E. The Brain's Default Mode Network. Annu. Rev. Neurosci.38, 433-447 (2015).

98. Mars, R. B. et al. On the relationship between the 'default mode network' and the 'social brain'. Front. Hum. Neurosci.6, 1-9 (2012).

99. Mitchell, J. P., Macrae, C. N. \& Banaji, M. R. Dissociable Medial Prefrontal Contributions to Judgments of Similar and Dissimilar Others. Neuron50, 655-663 (2006).

100. Wen, T., Mitchell, D. J. \& Duncan, J. The Functional Convergence and Heterogeneity of Social, Episodic, and Self-Referential Thought in the Default Mode Network. Cereb. Cortex30, 5915-5929 (2020).

101. Goldman-Rakic, P. S. Regional and cellular fractionation of working memory. Proc. Natl. Acad. Sci.93, 13473 LP - 13480 (1996).

102. Vogt, S. et al. Prefrontal involvement in imitation learning of hand actions: Effects of practice and expertise. Neuroimage37, 1371-1383 (2007).

103. Buccino, G., Binkofski, F. \& Riggio, L. The mirror neuron system and action recognition. Brain Lang.89, 370-376 (2004).

104. Tettamanti, M. et al. Listening to action-related sentences activates fronto-parietal motor circuits. J. Cogn. Neurosci.17, 273-281 (2005).

105. Binkofski, F. \& Buccino, G. The role of ventral premotor cortex in action execution and action understanding. J. Physiol. Paris99, 396-405 (2006). 
106. Petrides, M., Cadoret, G. \& Mackey, S. Orofacial somatomotor responses in the macaque monkey homologue of Broca's area. Nature435, 1235-1238 (2005).

107. Oldfield, R. C. The assessment and analysis of handedness: The Edinburgh inventory. Neuropsychologia9, 97-113 (1971).

108. R Core Team. R: A language and environment for statistical computing. (2020).

109. Taulu, S. \& Simola, J. Spatiotemporal signal space separation method for rejecting nearby interference in MEG measurements. Phys. Med. Biol.51, 1759-1768 (2006).

110. Delorme, A. \& Makeig, S. EEGLAB: an open source toolbox for analysis of single-trial EEG dynamics including independent component analysis. J. Neurosci. Methods134, 9-21 (2004).

111. Gross, J. et al. Dynamic imaging of coherent sources: Studying neural interactions in the human brain. Proc. Natl. Acad. Sci. U. S. A.98, 694-699 (2001).

112. Veen, B. D. Van, Drongelen, W. Van, Yuchtman, M. \& Suzuki, A. Localization of brain electrical activity via linearly constrained minimum variance spatial filtering. IEEE Trans. Biomed. Eng.44, 867-880 (1997).

113. Friston, K. J. et al. Statistical parametric maps in functional imaging: A general linear approach. Hum. Brain Mapp.2, 189-210 (1994).

114. Oostenveld, R., Fries, P., Maris, E. \& Schoffelen, J.-M. FieldTrip: Open Source Software for Advanced Analysis of MEG, EEG, and Invasive Electrophysiological Data. Comput. Intell. Neurosci.2011, 156869 (2011).

\section{Figures}




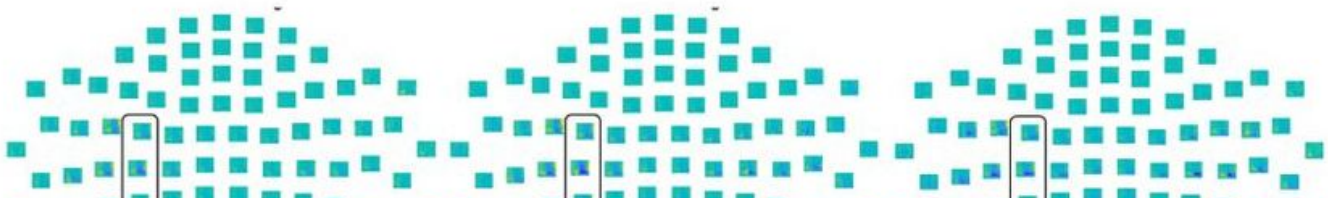

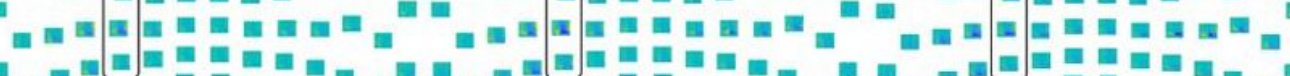

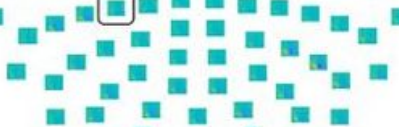

ㅁ.

뭄

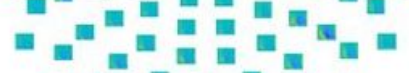

미믐

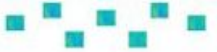

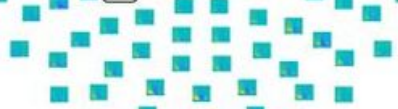

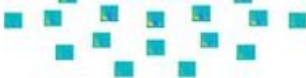
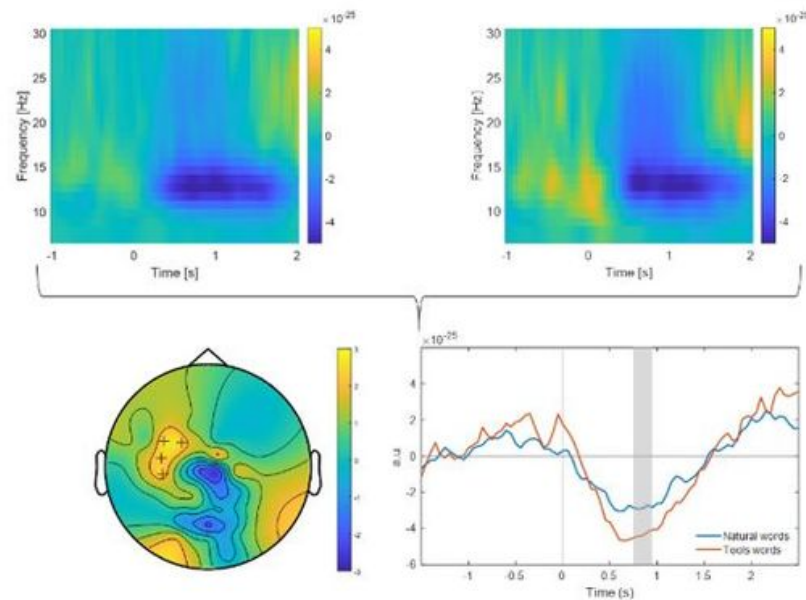

Figure 1

A. Time-frequency representations (TFR) for the different stimuli. Box indicates the contralateral motor area. B. Magnification of TFR in contralateral motor area. Note the beta pattern of desynchronization (reduction of power) and synchronization (increase of power) more evident in the case of tools stimuli C. Map of significant difference in beta band averaged over the time interval between 0.6 and $0.9 \mathrm{~s}$ for images and between 0.7 and $0.9 \mathrm{~s}$ for nouns, for each pair of stimulus type comparison (left: natural vs. tools images; right: natural vs. tools nouns). Asterisks indicate $p<0.01$, plus indicate $p<0.05$. D. Time course of beta band power modification for each stimulus type. Shadowed area indicates the time range where the difference was significant $(p<0.05)$. 


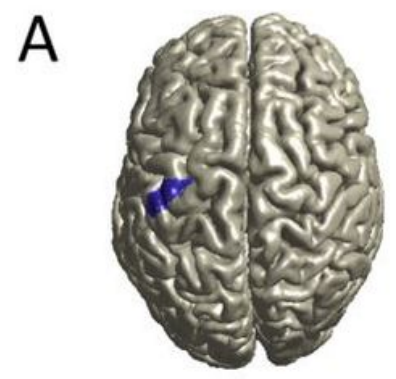

Natural images

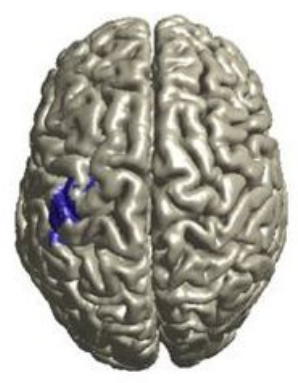

Tool images

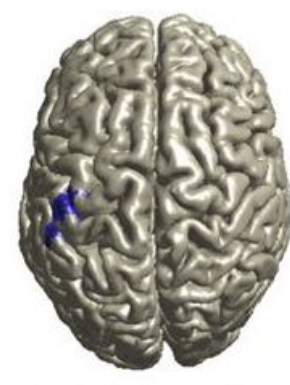

Natural words

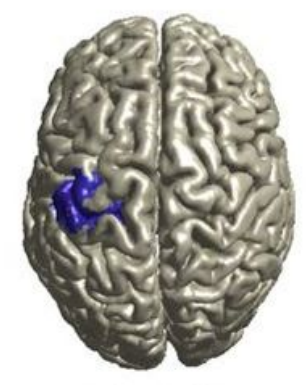

Tool words
B

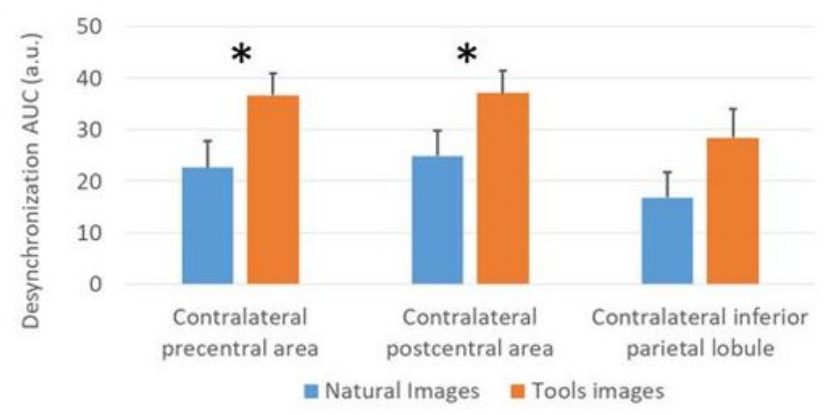

C

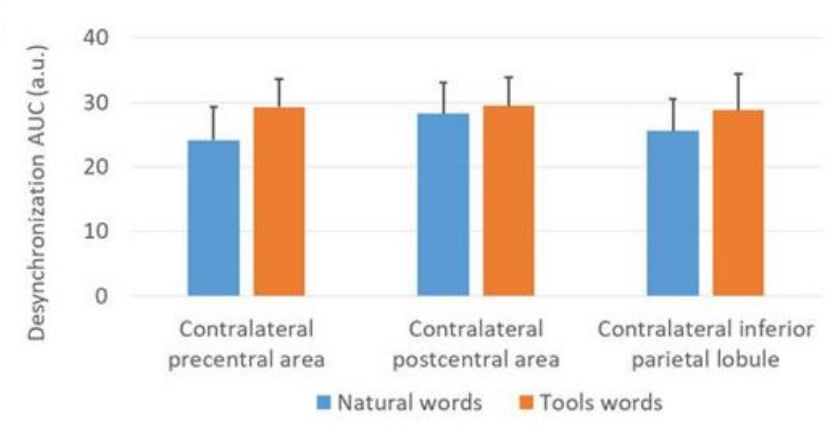

\section{Figure 2}

A: Source estimation projected onto the MNI template brain of grand-averaged power modulation obtained by contrasting -1.5 to $-0.5 \mathrm{~s}$ vs. 0.5 to $1.5 \mathrm{~s}$ with respect to the cue onset in $15-25 \mathrm{~Hz}$ band for each condition. For illustrative purpose, only values greater than $80 \%$ of the maximum are shown. B,C: Beta AUC values calculated in 0.5 to $1.5 \mathrm{~s}$ with respect to the cue onset for natural and tools images (B) and nouns (C) condition. Note that the natural stimuli values are smaller than tools stimuli in both images and nouns condition in all areas, confirming the main effect of Category. Asterisk indicates significant difference in t-tests. 


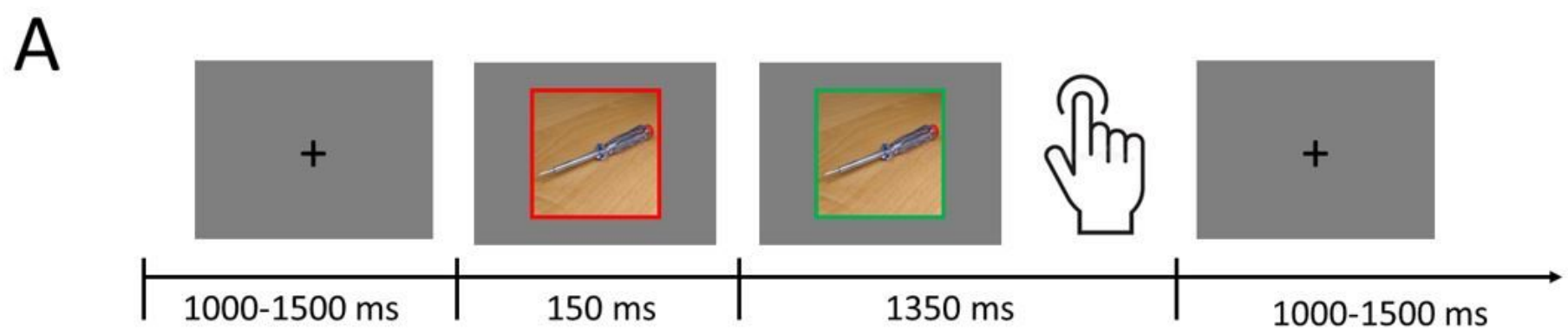

B

Tools

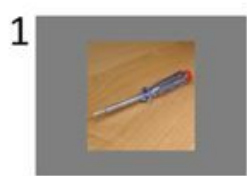

Image

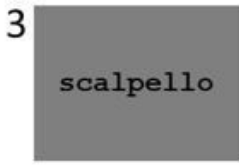

Noun

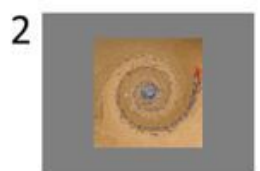

Scrambled image

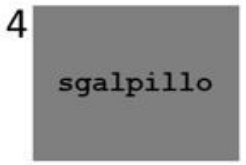

Pseudoword

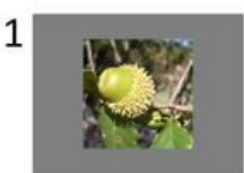

Image

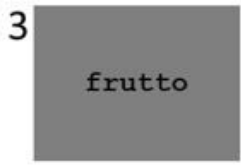

Noun
Natural object

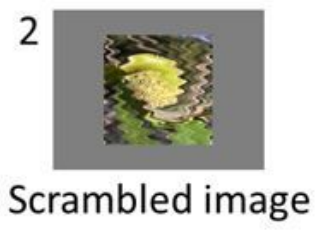

4

crutti

Pseudoword

\section{Figure 3}

Experimental procedure. A. Task timing: Participants were asked to fixate the centre of the screen placed in front of them. Each trial started with the presentation of the stimulus surrounded by a red frame. After $150 \mathrm{~ms}$ the frame turned green and the participants were allowed to respond. Participants were instructed to respond only if the stimulus referred to a real tool or to a real natural graspable object. The trial ended when participants provided their responses or after $1350 \mathrm{~ms}$ if no response was given. B. Stimuli examples: images (1), scrambled images (2), nouns (3) and pseudowords (4).

\section{Supplementary Files}

This is a list of supplementary files associated with this preprint. Click to download.

- NaturalToolssupplementary.docx 